\title{
Solute Analyse of Directional Solidification Microstructure in a Binary Alloy
}

\author{
Jinjun Tang ${ }^{1, \mathrm{a}}$, Mingan Zhou ${ }^{2, \mathrm{~b}}$ \\ ${ }^{1}$ Ningbo Sub-academy of the National Weapons Science Research Academy, Ningbo 315103, \\ China \\ ${ }^{2}$ Department of Mechanical Engineering, Quzhou College of Technology, Quzhou 324000, China \\ ajinjun.tang@163.com, ${ }^{b}$ renzheng_nb@163.com
}

Keywords: numerical simulation; Bridgman directional solidification; microsegregation; phase-field method; microstructure; pulling velocity;

\begin{abstract}
In this paper, the directional solidification microstructure of Bridgman system was simulated using phase-field method, and different calculated results were obtained with four pulling velocities. Transverse solute shows " $U$ " shape profiles. With the pulling velocity $\left(v_{p}\right)$ increasing, the span of " $U$ " figures decreases and the deepness of "U" figures decreases too. When the pulling velocity reaches $3.00 \mathrm{~cm} / \mathrm{s}$, the "U" figures disappear. With the supersaturation and the growing velocity increases, the directional microstructure changes from thin columnar crystals into much thinner columnar crystals. Transverse solute has a very little fluctuation around the initial concentration and the solute trapping phenomenon takes place.
\end{abstract}

\section{Introduction}

Alloy phase field models have been formulated by many scholars in the past fifteen years. Wheeler, Boettinger and McFadden introduced the phase field model [1, 2] for binary alloy firstly, which is called WBM model. J. J. Guo [3] simulats structure evolution of $\mathrm{Ti}_{55} \mathrm{Al}_{45}$ alloy during directional solidification. R. N. Costa Filho [4] steadies wavelength selection in two dimensions. C. W. Lan es al [5-7] study the morphological instability during directional solidification of a succinonitrile/acetone alloy. H. M. Singer [8] determines morphology diagrams in two and three dimensions for two different phase-field models. Mathis Plapp [9] investigates three-dimensional phase-field simulation of directional solidification. In this paper, different microstructures are obtained by changing the pulling velocities, and concentration field and microsegregation are also discussed.

\section{The phase-field model}

Phase field method is an effective way to solve the problem of complicated phase transformation. According to material thermodynamics, the free energy density of pure component $A$ and $B, f_{A}(\Phi, T)$ and $f_{B}(\Phi, T)$, are given by

$$
f_{A}(\Phi, T)=W_{A} g(\Phi)+L_{A} \frac{T_{m}^{A}-T}{T_{m}^{A}} p(\Phi), \quad f_{B}(\Phi, T)=W_{B} g(\Phi)+L_{B} \frac{T_{m}^{B}-T}{T_{m}^{B}} p(\Phi)
$$

$W_{A}$ and $W_{B}$ are the energy barriers of the pure component $A$ and $B$, and $T_{M}^{A}$ and $T_{M}^{B}$ are the melting points of pure materials $\mathrm{A}$ and $\mathrm{B}, L^{A}$ and $L^{B}$ are the latent heat of pure materials $\mathrm{A}$ and $\mathrm{B}$ and $\mathrm{g}(\Phi)$ and $\mathrm{p}(\Phi)$ are the functions of phase-field variable, which are given by

$$
g(\Phi)=\Phi^{2}(1-\Phi)^{2}, p(\Phi)=\Phi^{3}\left(10-15 \Phi+6 \Phi^{2}\right)
$$

The density of system free energy, $f(c, \Phi, T)$, is given by

$$
f(c, \Phi, T)=(1-c) f_{A}(\Phi, T)+c f_{B}(\Phi, T)+\frac{R_{g} T}{v_{m}}[c \ln c+(1-c) \ln (1-c)]+c(1-c)\left\{\Omega_{L}[1-p(\Phi)]+\Omega_{S} p(\Phi)\right\}
$$

where $R_{g}$ is gas constant and $v_{m}$ is molar volume, $\Omega_{L}$ and $\Omega_{S}$ are the parameters of regular 
solution, which denote ideal solution when they are both equal zero.In our simulations, Ni-Cu alloy is used, which is an ideal solution, so $\Omega_{L}$ and $\Omega_{S}$ are both equal zero. The system free energy, $F$, can be obtained by a volume integral, given by

$$
F=\int_{V}\left[f(c, \Phi, T)+\frac{\varepsilon^{2}}{2}|\nabla \Phi|^{2}\right] d V
$$

where $\varepsilon$ is gradient energy coefficient, which is given by

$$
\varepsilon=\varepsilon_{0}(1+r \cos (k \theta)) \quad \varepsilon_{0}^{2}=6 \sigma^{A} \delta^{A}
$$

where $\varepsilon_{0}$ is parameters of phase field, $\gamma$ is the intensity of anisotropism, $k$ is mode number, and $\theta$ is the included angle from coordinate axis to main arbor of growth. According to Landau theory, there are equations below

$$
\begin{aligned}
& \frac{\partial \Phi}{\partial t}=-M_{\Phi} \frac{\partial F}{\partial \Phi} \\
& \frac{\partial c}{\partial t}=\nabla \cdot\left[M_{c} c(1-c) \nabla\left(\frac{\partial F}{\partial c}\right)\right]
\end{aligned}
$$

$M_{\Phi}$ and $M_{C}$ are phase-field kinetic parameter, $M_{A}, M_{B}, W_{A}$ and $W_{B}$, are parameters of the phase field model, given by

$$
\begin{aligned}
& M_{C}=\frac{D_{S}+p(\Phi)\left(D_{L}-D_{S}\right)}{R_{g} T / v_{m}} \quad M_{\Phi}=(1-c) M_{A}+c M_{B} \\
& M_{A}=\frac{T_{m}^{A} \beta^{A}}{6 L^{A} \delta^{A}}, M_{B}=\frac{T_{m}^{B} \beta^{B}}{6 L^{B} \delta^{B}} \quad W_{A}=\frac{3 \sigma^{A}}{\delta^{A}}, W_{B}=\frac{3 \sigma^{B}}{\delta^{B}}
\end{aligned}
$$

$\beta^{A}$ and $\beta^{B}$ are the kinetic coefficient of pure materials $\mathrm{A}$ and $\mathrm{B}$, and $\delta^{A}$ and $\delta^{B}$ are the interface thickness of pure materials $\mathrm{A}$ and $\mathrm{B}$ and $\sigma^{A}$ and $\sigma^{B}$ are the interfacial energy of pure materials $\mathrm{A}$ and B. In the Bridgman Directional solidification system, the equation (6) and (7) are recast into a moving frame with constant speed $V_{P}$, given by

$$
\begin{aligned}
& \frac{\partial \Phi}{\partial t}-V_{P} \frac{\partial \Phi}{\partial y}=-M_{\Phi} \frac{\partial F}{\partial \Phi} \\
& \frac{\partial c}{\partial t}-V_{P} \frac{\partial c}{\partial y}=\nabla \cdot\left[M_{c} c(1-c) \nabla\left(\frac{\partial F}{\partial c}\right)\right]
\end{aligned}
$$

From the observer point of view, the system can be regarded as a domain having continuous feeding from the top and flowing out from the bottom. The horizontal heat transfer is ignored and only the vertical one dimension heat conduction is considered. This approximation is tenable only in a very high temperature gradient. The system temperature is given by

$$
T(y)=T_{B}+G y-G V_{P} \quad G=\frac{T_{H}-T_{B}}{H}
$$

$G$ is temperature gradient and equals $800 \mathrm{~K} / \mathrm{cm}$ in this paper, $T_{H}$ and $T_{B}$ are the temperatures of the highest and lowest ends and $H$ is the highness of the calculational zone. In this simulation below, the pulling velocity is set $0.06 \mathrm{~cm} / \mathrm{s}, 0.40 \mathrm{~cm} / \mathrm{s}, 1.00 \mathrm{~cm} / \mathrm{s}$, and $3.00 \mathrm{~cm} / \mathrm{s}$ and different microstructures are obtained. The Zero-Neumann boundary conditions for $\Phi$ and $\mathrm{C}$ are imposed at the boundaries of the computational domain, and described by $\frac{\partial \Phi}{\partial n}=0 ; \frac{\partial c}{\partial n}=0$.

\section{Results and discussion}

When the pulling velocity reaches $3.00 \mathrm{~cm} / \mathrm{s}$ as shown in Fig.1, the microsegregation decreases further. The solute boundary in front of interface is much thinner. The disturbance is unconspicuous in the condition of bigger supercooling and quicker growing velocity. The growth of solid phase is planar interface manner, and there is no obvious grain boundary between columnar crystals. The solute difference between fluid and solid phase decreases further. As shown in Fig.1 (a), the microsegregation is the lowest, and reaches about 1.03. As shown in Table.1, the highest composition is 0.420 , and the lowest composition is 0.407 . Thereby, increasing the pulling velocity can change thinner columnar crystals into the crystals of no grain boundary when the absolute 
stability condition of high rate $\left(V_{A}\right)$ is reached.
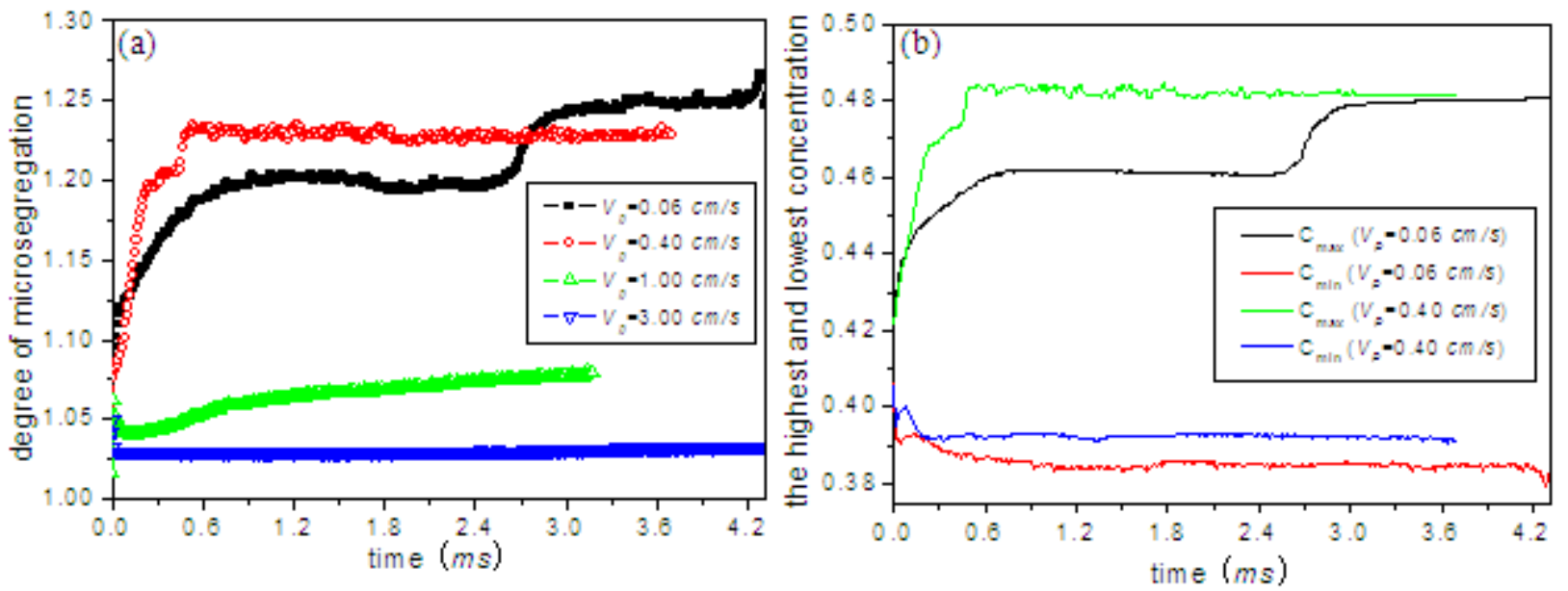

Fig.1 Microsegregation and the highest and lowest concentration versus time
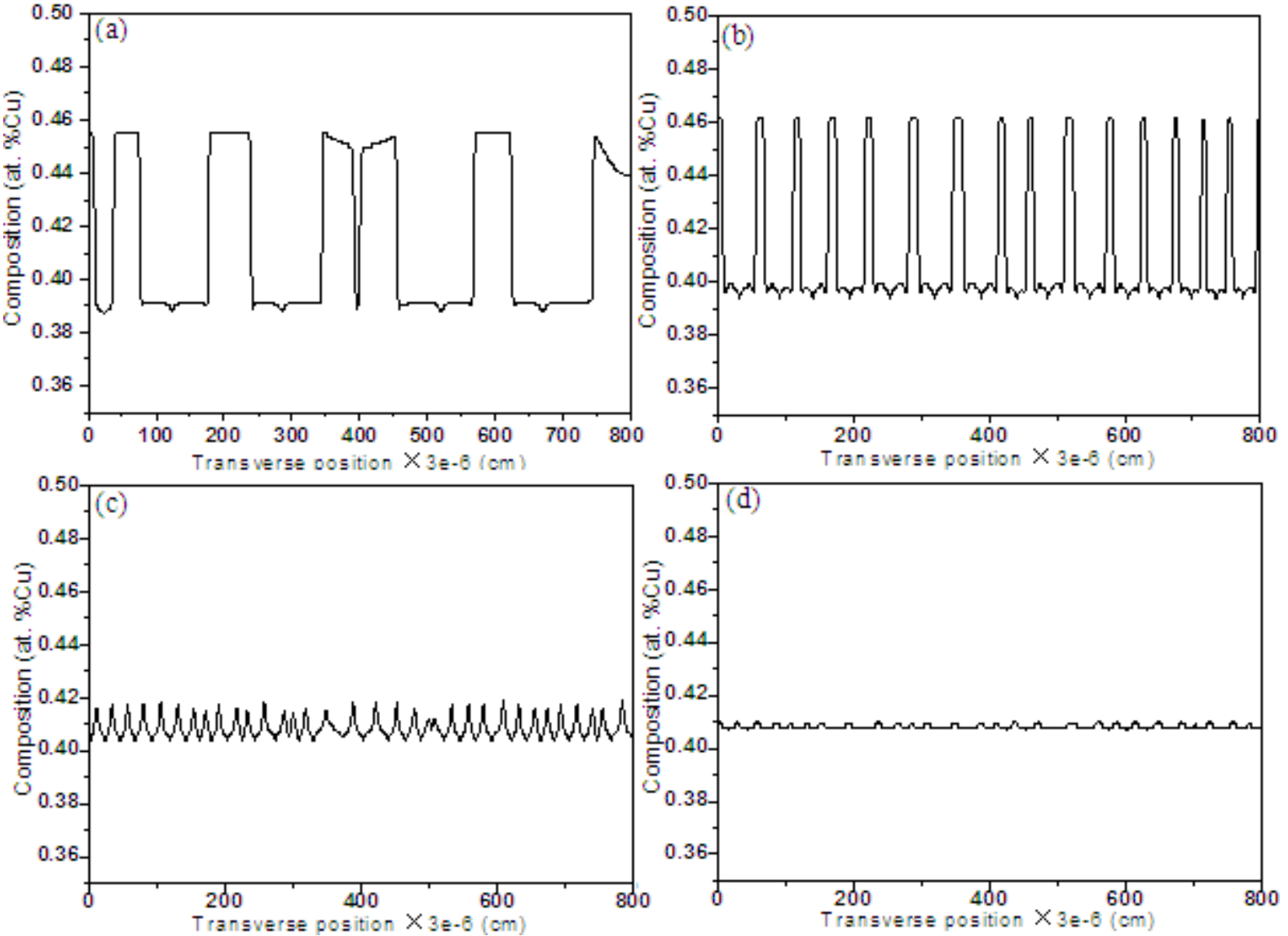

Fig.2 Transverse solute profiles of different pulling velocity $\left(v_{P}\right)$ toward steady state solidification (a) $v_{P}=0.06 \mathrm{~cm} / \mathrm{s}$ (b) $v_{P}=0.40 \mathrm{~cm} / \mathrm{s} \mathrm{(c)} v_{P}=1.00 \mathrm{~cm} / \mathrm{s}(\mathrm{d}) v_{P}=3.00 \mathrm{~cm} / \mathrm{s}$

Table.1 Calculated results of different pulling velocity $\left(v_{P}\right)$

\begin{tabular}{|l|l|l|l|l|}
\hline pulling velocity $\left(v_{P}\right) \quad / \mathrm{cm} / \mathrm{s}$ & 0.06 & 0.40 & 1.00 & 3.00 \\
\hline The bottom temperature / K & 1575.5 & 1575 & 1568 & 1564 \\
\hline The highest composition / at.\%Cu & 0.481 & 0.484 & 0.434 & 0.420 \\
\hline The lowest composition / at.\%Cu & 0.379 & 0.391 & 0.403 & 0.407 \\
\hline The biggest degree of microsegregation & 1.268 & 1.234 & 1.079 & 1.032 \\
\hline The average space length of columnar crystals / cm & $0.511 \mathrm{e}-3$ & $0.178 \mathrm{e}-3$ & $0.075 \mathrm{e}-3$ & - \\
\hline
\end{tabular}

In order to observe solute trapping phenomenon, transverse solute profiles of different pulling velocities are given in Fig.2. As showed in Fig.6, the solute profiles of columnar crystals look like a "U" shape. The span of the "U" profile reflects the columnar crystals thickness and the deepness of the " $U$ " profile reflects the microsegregation of calculational domain concerned. The distance 
between the centers of "U" profile is the space length of columnar crystals. Solute is low in the center of the columnar crystal and solute is higher between the columnar crystals. With the pulling velocity increasing, the number of " $U$ " figures increases gradually and the spans decrease gradually. Accordingly, the solute in the center of the columnar crystals increases gradually and approache to the initial concentration. The solute between columnar crystals decreases gradually. Thereby, the wave height of " $U$ " profiles decreases and the microsegregation decreases. When the pulling velocity reaches $3.00 \mathrm{~cm} / \mathrm{s}$, "U" waves disappears. Transverse solute has a very little fluctuation around the initial concentration. The degree of systematic microsegregation is very low and approaches to 1 .

\section{Conclusion}

The pulling velocity $\left(v_{p}\right)$ also plays an important role on microsegregation. If $v_{p}$ is smaller than $V_{A}$, microsegregation increases with the pulling velocity $\left(v_{p}\right)$ increasing. Once $v_{p}$ greater than $V_{A}$, microsegregation decreases with the pulling velocity $\left(v_{p}\right)$ increasing and approaches gradually to 1 . Transverse solute shows "U" shape profiles. With the pulling velocity $\left(v_{p}\right)$ increasing, the span of "U" figures decreases and the deepness of " $U$ " figures decreases too. When the pulling velocity reaches $3.00 \mathrm{~cm} / \mathrm{s}$, the "U" figures disappear. Transverse solute has a very little fluctuation around the initial concentration and the solute trapping phenomenon takes place.

\section{References}

[1] A. A. Wheeler, W. J. Boettinger and G. B. McFadden. Phys. Rev. A. 45 (1992) 7424-7439.

[2] A. A. Wheeler, W. J. Boettinger and G. B. McFadden. Phys. Rev. E. 47 (1993) 1893-1909.

[3] J. J. Guo, X. Z. Li, Y. Q. Su, S. P. Wu, B. S. Li and H. Z. Fu. Rare Metal Materials and Engineering. 33 (2004) 195.

[4] R. N. Costa Filho, J. M. Kosterlitz and Enzo Granato. Physica A. 354 (2005) 333.

[5] C. W. Lan and Y. C. Chang. J. Cryst. Growth. 250 (2003) 525.

[6] C. W. Lan, C. J. Shih and M. H. Lee. Acta. Mater. 53 (2005) 2285.

[7] C. W. Lan, M. H. Lee, M. H. Chuang, C. J. Shih. J. Cryst. Growth. 295 (2006) 202.

[8] H. M. Singer, I. Singer-Loginova, J. H. Bilgram and G. Amberg. J. Cryst. Growth. 296 (2006)

58.

[9] Mathis Plapp. J. Cryst. Growth. 303 (2007) 49. 